\title{
Relationship between Clozapine-Induced Electroencephalogram Abnormalities and Serum Concentration of Clozapine in Japanese Patients with Treatment-Resistant Schizophrenia
}

\author{
Yuka Sugawara Kikuchi ${ }^{\bowtie}$, Takashi Kanbayashi, and Tetsuo Shimizu \\ Department of Neuropsychiatry, Akita University Graduate School of Medicine, Akita, Japan
}

Objective The objective of this study was to investigate the relationship between the serum concentration of clozapine (C-CLZ), Ndesmethylclozapine (N-CLZ) and the daily dose of CLZ (D-CLZ), and the relationships among CLZ and electroencephalogram (EEG) abnormalities.

Methods Twenty-eight patients were recruited to this study, but 8 patients were excluded because clozapine was discontinued before the post-treatment measurement of EEG or C-CLZ. Ultimately, 20 patients ( 6 men, 14 women) with an average age of 36 years were enrolled. The subjects were divided into EEG normal and abnormal groups. C-CLZ and N-CLZ were measured at 4, 12, 26, and 52 weeks after initiating CLZ administration.

Results All patients had normal baseline EEG signals, and 8 patients showed EEG abnormalities later. There were significant correlations between C-CLZ and D-CLZ, and between N-CLZ and D-CLZ. The C-CLZ/D-CLZ, N-CLZ/D-CLZ, and C-CLZ/N-CLZ ratio were not significantly different between the EEG normal and EEG abnormal groups. The EEG abnormal group had significant higher proportion of patients with high intra-individual variability in their C-CLZ/D-CLZ ratio.

Conclusion There is no relationship between C-CLZ and EEG abnormalities. However, patients with high intra-individual variability in their C-CLZ/D-CLZ ratio had greater possibility of exhibiting EEG abnormalities.

Psychiatry Investig 2019;16(4):279-284

Key Words Serum clozapine concentration, Electroencephalography abnormalities, Treatment-resistant schizophrenia.

\section{INTRODUCTION}

Clozapine (CLZ) is effective against treatment-resistant schizophrenia (TRS), and was introduced to Japan in 2009. In Japan, all physicians who prescribe CLZ, and their patients, must be registered with the Clozaril Patient Monitoring Service. In addition, CLZ administration is limited to patients with TRS who exhibit defined poor response or satisfy poor tolerance criteria.

It is known that CLZ induces serious side effects, such as agranulocytosis and seizures. Electroencephalogram (EEG) abnormalities are even more common. It has been reported

\footnotetext{
Received: September 22, 2018 Accepted: December 16, 2018

$\triangle$ Correspondence: Yuka Sugawara Kikuchi, MD, PhD

Department of Neuropsychiatry, Akita University Graduate School of Medicine, 1-1-1 Hondo, Akita 010-8543, Japan

Tel: +81-18-884-6122, Fax: +81-18-884-6445

E-mail: yuka@med.akita-u.ac.jp

(c) This is an Open Access article distributed under the terms of the Creative Commons Attribution Non-Commercial License (https://creativecommons.org/licenses/bync/4.0) which permits unrestricted non-commercial use, distribution, and reproduction in any medium, provided the original work is properly cited.
}

that CLZ-induced EEG abnormalities occur in a dose-dependent manner, and correlate with the serum concentration of CLZ (C-CLZ) ${ }^{1-4}$ However, contradictory results have also been reported. ${ }^{5}$ There is no convincing evidence that C-CLZ is related to EEG abnormalities. However, the studies mentioned above were performed on Caucasian patients. Ethnicity has been shown to influence clozapine pharmacokinetics. ${ }^{6}$ For example, lower daily clozapine doses are needed to achieve comparable blood levels in Asians compared to Caucasians; ${ }^{7}$ C-CLZ has been reported to be significantly lower in Korean patients than in Caucasian patients. ${ }^{8}$

$\mathrm{N}$-desmethylclozapine ( $\mathrm{N}-\mathrm{CLZ})$ is a major active CLZ metabolite, mainly produced by oxidative metabolism of $\mathrm{CY}$ P1A2. CLZ and N-CLZ have opposing actions on the cholinergic system. With regard to cognition, the C-CLZ to N-CLZ $(\mathrm{C} / \mathrm{N})$ ratio is a strong predictor of working memory performance in patients with schizophrenia, although C-CLZ and $\mathrm{N}-\mathrm{CLZ}$ alone show no association. ${ }^{9}$ With regard to EEG abnormalities, the usability of the $\mathrm{C} / \mathrm{N}$ ratio is unclear.

We investigated the following in Japanese patients with 
schizophrenia: 1) the relationship between C-CLZ, N-CLZ, and the daily dose of CLZ (D-CLZ), and 2) the relationship among CLZ and EEG abnormalities.

\section{METHODS}

\section{Subjects and demographic profiles}

Twenty-eight patients, who provided written informed consent, were recruited into this study. The study was approved by our Institutional Review Board (IRB 746). This study was conducted according to the principles expressed in the Declaration of Helsinki. The subjects were all consecutively hospitalized patients with TRS. All patients met the criteria of poor response or poor tolerance. Poor response is defined as failure to respond to a sufficient term (at least 4 weeks) of treatment with a sufficient dose of at least two well-tolerated antipsychotics [including at least one atypical antipsychotic (such as risperidone, perospirone, olanzapine, quetiapine, and aripiprazole) at over $600 \mathrm{mg} / \mathrm{d}$ chlorpromazine equivalent]. Poor tolerance is defined as the failure to adequately respond to monotherapy with at least two atypical antipsychotics, such as risperidone, perospirone, olanzapine, quetiapine, and aripiprazole, due to a failure to increase the dose to a necessary level for any of the following reasons: occurrence or worsening of moderate or more severe tardive dyskinesia, tardive dystonia, or other tardive extrapyramidal symptoms; occurrence of uncontrolled parkinsonian symptoms, akathisia, or acute dystonia. No patient had received CLZ before, or had a history of seizure or brain injury. Five patients were excluded because CLZ was discontinued before the post-treatment measurement of EEG or C-CLZ because of cardiomyopathy, agranulocytosis, leucopenia, and pure red cell aplasia. Three more patients were excluded because their serum concentrations of CLZ were not obtained. Ultimately, 20 patients (6 men, 14 women), who were on average 36 years old, were enrolled. Administration of CLZ (Novartis International AG, Basel, Switzerland) was initiated at a dose of $12.5 \mathrm{mg} / \mathrm{d}$ on the first day, and was increased to $25 \mathrm{mg} / \mathrm{d}$ on the second day. On the third day and thereafter, the daily dose was increased slowly, in increments of $25 \mathrm{mg}$, to achieve a dose of up to $200 \mathrm{mg} / \mathrm{d}$ over 3 weeks. The dose was increased according to symptoms; however, an interval of at least 4 days was maintained between increments of doses up to $100 \mathrm{mg}$. CLZ was administered once or twice daily at 9:00 and/or 21:00. The maximum dose was $600 \mathrm{mg} / \mathrm{d}$, which is well-defined in Japan. In principle, the duration of hospitalization was defined as 18 weeks after initiating clozapine administration. However, given the following criteria were met, the patients were discharged before 18 weeks: desire for discharge, achievement of the maintenance dosage, at least 3 weeks of hospitalization, improve- ment of clinical symptoms, and confirmation of safety.

\section{EEG recording}

The EEG recordings were performed using the International 10-20 system and a 21-channel monitor. All records were assessed with the patient's history and medications blinded. The EEG recordings were obtained every 4 weeks, several days prior to CLZ treatment. After the occurrence of EEG abnormalities, extra measurements of EEG were performed every 2 weeks.

\section{Measurement of the serum concentration of CLZ}

All patients received CLZ for at least 4 weeks. Plasma concentrations of both CLZ and N-CLZ are known to reach steady-state levels by 4 weeks after repeated oral administration. The levels of C-CLZ and N-CLZ were measured at 8:30, and, subsequently, $4,12,26$, and 52 weeks after initiating CLZ administration.

Blood samples were stored at $-20^{\circ} \mathrm{C}$ until assayed. C-CLZ and N-CLZ were measured using high-purity high-performance liquid chromatography (HPLC) with ultraviolet (UV) detection. The HPLC system consisted of a Shimadzu Nexera and a Shimadzu Workstation LC solution chromatography integrator (Kyoto, Japan). The peaks were detected by a UV detector, at a wavelength of $254 \mathrm{~nm}$. The peak areas were used for the quantification of C-CLZ and N-CLZ.

\section{Data analysis}

The subjects were divided into EEG normal and abnormal groups. The correlations between C-CLZ and D-CLZ, and between N-CLZ and D-CLZ were evaluated for each group using Spearman's rank-correlation coefficient. The C-CLZ per D-CLZ (C/D), N-CLZ per D-CLZ (N/D), and C/N ratio were compared between the two groups using Student's t-tests. The threshold of significance for all tests was set at $5 \%$.

\section{RESULTS}

All patients had normal baseline EEG, and 8 patients (40\%) showed EEG abnormalities later ( 1 patient at 4 weeks; 5 patients at 8 weeks; 1 patient at 9 weeks; 1 patient at 52 weeks). The EEG findings were spike and slow wave (7 patients, $88 \%$ ), theta (5 patients, 63\%), and delta (3 patients, 38\%). No patients withdrew from this study because of the appearance of EEG abnormalities. The patient's characteristics are outlined in Table 1. Seventy-one serum levels were measured in total. There was a significant correlation between C-CLZ and D-CLZ (EEG normal: rs 0.58, p<0.01; EEG abnormal: rs $0.46, \mathrm{p}=0.01$ ), and between N-CLZ and D-CLZ (EEG normal: rs 0.53, p<0.01; EEG abnormal: rs 0.48, $\mathrm{p}=0.01$ ) (Table 2). The 
Table 1. Patients' characteristics

\begin{tabular}{|c|c|c|c|c|c|c|}
\hline & No & Age & Sex & $\begin{array}{c}\text { Illness duration before CLZ } \\
\text { treatment (years) }\end{array}$ & Comorbidities & Concomitant medications \\
\hline \multirow[t]{8}{*}{ EEG abnormal } & 1 & 48 & $\mathrm{~F}$ & 14 & None & None \\
\hline & 2 & 25 & $\mathrm{~F}$ & 7 & None & None \\
\hline & 3 & 22 & $\mathrm{~F}$ & 9 & None & Diazepam \\
\hline & 4 & 24 & $\mathrm{~F}$ & 4 & None & Brotizolam \\
\hline & 5 & 37 & $\mathrm{~F}$ & 17 & None & Brotizolam \\
\hline & 6 & 22 & $\mathrm{~F}$ & 2 & None & Diazepam \\
\hline & 7 & 22 & $\mathrm{~F}$ & 2 & None & Diazepam \\
\hline & 8 & 17 & M & 1 & None & None \\
\hline \multirow[t]{12}{*}{ EEG normal } & 1 & 34 & $\mathrm{~F}$ & 17 & None & None \\
\hline & 2 & 64 & $\mathrm{~F}$ & 40 & Hypertension & Diazepam \\
\hline & 3 & 36 & M & 20 & None & Brotizolam \\
\hline & 4 & 40 & $\mathrm{~F}$ & 8 & None & Diazepam \\
\hline & 5 & 65 & M & 25 & Orthostatic hypotension & Triazolam \\
\hline & 6 & 48 & $\mathrm{~F}$ & 19 & None & Brotizolam \\
\hline & 7 & 33 & $\mathrm{M}$ & 9 & None & None \\
\hline & 8 & 27 & $\mathrm{~F}$ & 4 & None & Brotizolam \\
\hline & 9 & 20 & $\mathrm{~F}$ & 5 & None & Lorazepam \\
\hline & 10 & 47 & $\mathrm{M}$ & 20 & None & None \\
\hline & 11 & 39 & $\mathrm{M}$ & 12 & None & None \\
\hline & 12 & 43 & $\mathrm{~F}$ & 10 & None & Diazepam \\
\hline
\end{tabular}

CLZ: clozapine, EEG: electroencephalogram, F: female, M: male

Table 2. Correlation coefficients between C-CLZ and D-CLZ, and between N-CLZ and D-CLZ in the EEG normal and EEG abnormal groups

\begin{tabular}{ccccc}
\hline & Average & SD & rs with D-CLZ & p value \\
\hline EEG normal group & & & & \\
D-CLZ (mg) & 300.0 & 153.6 & & \\
C-CLZ (ng/mL) & 581.4 & 358.2 & 0.58 & $<0.01$ \\
N-CLZ (ng/mL) & 329.0 & 219.7 & 0.53 & $<0.01$ \\
EEG abnormal group & & & & \\
D-CLZ (mg) & 248.3 & 90.9 & & \\
C-CLZ (ng/mL) & 444.7 & 319.0 & 0.46 & 0.01 \\
N-CLZ (ng/mL) & 232.4 & 124.1 & 0.48 & 0.01 \\
\hline
\end{tabular}

C-CLZ: serum concentration of clozapine, N-CLZ: N-desmethylclozapine, D-CLZ: daily dose of clozapine, rs: Spearman's Rank Correlation Coefficient, SD: standard deviation, EEG: electroencephalogram

$\mathrm{C} / \mathrm{D}, \mathrm{N} / \mathrm{D}$, and $\mathrm{C} / \mathrm{N}$ ratio were not significantly different between the EEG normal and EEG abnormal groups (Table 3). Figure 1 shows the time course of C/D in all patients from both groups. The average $\mathrm{C} / \mathrm{D}$ ratio was 2.0. The percentage of patients whose $\mathrm{C} / \mathrm{D}$ ratio was greater than 2 was $50 \%$ in the EEG abnormal group (4 out of 8 patients) and $8 \%$ in the EEG normal group (1 out of 12 patients). There was a significant
Table 3. The differences in the $C / D, N / D$, and $C / N$ ratios between the EEG normal and EEG abnormal groups

\begin{tabular}{lccc}
\hline & $\begin{array}{c}\text { EEG normal } \\
\text { group }\end{array}$ & $\begin{array}{c}\text { EEG abnormal } \\
\text { group }\end{array}$ & p value \\
\hline C/D (SD) & $2.10(1.22)$ & $1.84(1.12)$ & 0.35 \\
N/D (SD) & $1.20(0.82)$ & $1.00(0.59)$ & 0.26 \\
C/N ratio (SD) & $1.90(0.69)$ & $1.98(0.80)$ & 0.67 \\
\hline
\end{tabular}

C/D: serum concentration of clozapine/daily dose of clozapine, N/ $\mathrm{D}$ : N-desmethylclozapine/daily dose of clozapine, $\mathrm{C} / \mathrm{N}$ ratio: serum concentration of clozapine/N-desmethylclozapine, SD: standard deviation, EEG: electroencephalogram

difference between the groups (chi-square test, $\mathrm{p}<0.05$ ).

\section{DISCUSSION}

In this study, there were significant correlations between $C$ CLZ and D-CLZ, and between N-CLZ and D-CLZ. The mean D-CLZ, C-CLZ, and N-CLZ in this Japanese study are similar to previous reports. ${ }^{10-15}$ However, the correlation coefficients were only moderate. These results are similar to those from the study by Chang et al., ${ }^{10}$ which reported that the correlation coefficient between plasma concentrations and DCLZ was moderate ( $r=0.590)$. The authors explained the low coefficients by the large inter-patient variations in plasma con- 


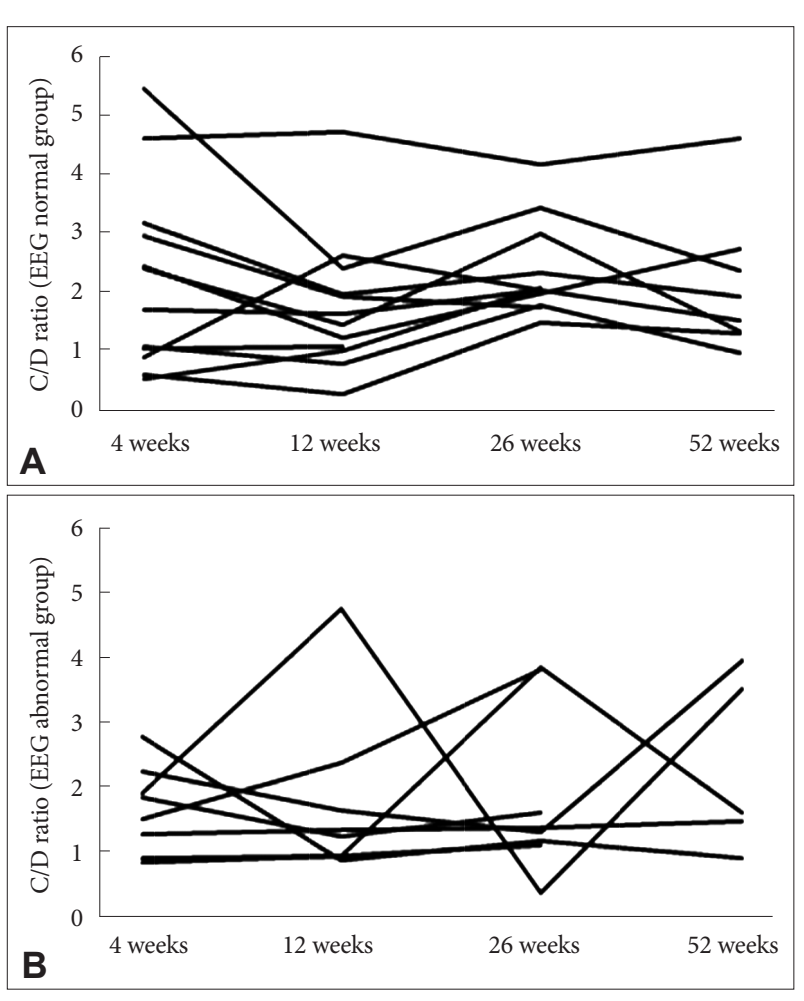

Figure 1. The time course of the $C / D$ ratio for each patient from both groups. A: C/D ratio of the EEG normal group, B: C/D ratio of the EEG abnormal group. C/D: serum concentration of clozapine/ daily dose of clozapine, EEG: electroencephalogram.

centrations of CLZ at the same dosage. Many studies have shown that variables such as sex, smoking, age, body weight, caffeine intake, and concomitant use of drugs may affect CYP1A2 activity. ${ }^{3,16,17-25}$ However, contradictory evidence regarding the association between plasma CLZ concentrations with sex and age also exists. ${ }^{14,19,26-28}$ In addition, Piwowarska et al. ${ }^{12}$ analyzed the C-CLZ and its metabolites using a repeated measures multivariate analysis of variance. They reported a significant correlation between D-CLZ and C-CLZ with a low correlation coefficient $(0.2674, \mathrm{p}<0.05)$, but the relationship between C-CLZ and D-CLZ was not linear at all doses. The C-CLZ in serum when the dose of the drug is higher and exceeds $500 \mathrm{mg}$ might be explained by the inhibition of CYP1A2 by the drug.

Among typical and atypical antipsychotics, CLZ is the drug most strongly associated with EEG abnormalities (CLZ: 47.1\%, olanzapine: $38.5 \%$, risperidone: $28.0 \%$, typical neuroleptics: 14.5\%, quetiapine: $0.0 \%){ }^{29}$ The EEG abnormalities reported to be associated with CLZ treatment range from $16 \%$ to $74 \%$.,30 Haring et al. ${ }^{13}$ described EEG abnormalities in $53 \%$ of patients, and reported that these were dependent on the plasma levels of CLZ. The authors determined that the dose was not statistically related to the EEG abnormalities. Subsequently, it has been reported that CLZ-induced EEG abnormalities occur in a dose-dependent manner, and are correlated with serum levels of CLZ. ${ }^{1,2,4,13}$ However, Goyal et al. ${ }^{5}$ reported EEG abnormalities in $61.9 \%$ of patients receiving $100 \mathrm{mg}$ or less of CLZ. Freudenreich et al. ${ }^{1}$ reported that $13 \%$ of patients develop spikes with no relationship to the dose of CLZ or CCLZ.

The plasma concentration of N-CLZ, a known major metabolite of CLZ, can vary from $20 \%$ to $150 \%$ of C-CLZ. ${ }^{31}$ The $\mathrm{C} / \mathrm{N}$ ratio is a strong predictor of working memory and cognitive performances in patients with schizophrenia, even though the concentrations of C-CLZ and N-CLZ alone are not, which is likely because of the opposing effects of CLZ and N-CLZ.9.15 The $\mathrm{C} / \mathrm{N}$ ratio has been suggested to be a valuable predictor of response to CLZ in patients with TRS. ${ }^{32}$ Anderson et al. ${ }^{33}$ reported that the $\mathrm{C} / \mathrm{N}$ ratio may be a significant factor in determining the increased incidence of weight gain and dysmetabolic profiles in female patients. The $\mathrm{C} / \mathrm{N}$ ratio may possibly represent a useful index for the side-effects of CLZ. However, in this study, the $\mathrm{C} / \mathrm{D}, \mathrm{N} / \mathrm{D}$, and $\mathrm{C} / \mathrm{N}$ ratios were not significantly different between the EEG normal and EEG abnormal groups. Therefore, the $\mathrm{C} / \mathrm{N}$ ratio contributes little to $\mathrm{EEG}$ abnormalities, which contrasts with its relationship with working memory and cognitive function. In addition, both the $\mathrm{C} /$ $\mathrm{D}$ and N/D ratios were not significantly different between the EEG normal and EEG abnormal groups. Our findings suggest that there is no relationship between C-CLZ and EEG abnormalities. Considering the intra-individual variability of the $\mathrm{C} / \mathrm{D}$ ratio, the percentage of patients whose $\mathrm{C} / \mathrm{D}$ ratio was greater than 2 was $50 \%$ in the EEG abnormal group and $8 \%$ in the EEG normal group. There was a significant difference between the groups $(\mathrm{p}<0.05)$. These results suggest that the patients with high intra-individual variability in the C/D ratio are predisposed to EEG abnormalities. To our knowledge, there are no reports regarding the relationship between the intra-individual variability of the C/D ratio and EEG abnormalities. Rajkumar et al. ${ }^{34}$ demonstrated a 30 -fold inter-individual variability among the participants, with their C/D ratio ranging from 0.28 to 8.29 . Several studies have shown an influence of variables such as sex, smoking, age, body, caffeine intake, and the concomitant use of drugs on CYP1A2 activity. ${ }^{3,16,17-25}$ Piwowarska et al. ${ }^{12}$ showed that the mean C-CLZ indicates a proportional increase in the drug concentrations in relation to the used doses. Even the C-CLZ measurements in one patient, who had measurements taken 11 times within 13 months, yielded various values for CLZ at the same dose during treatment. ${ }^{12}$

A limitation of this study was the small sample size. Therefore, it is necessary to conduct further studies including larger sample populations to confirm the association between the intra-individual variability of $\mathrm{C} / \mathrm{D}$ and other side-effects of CLZ. 
In conclusion, there are some reports that EEG abnormalities are associated with the dose of CLZ or C-CLZ. However, evidence to the contrary also exists. We demonstrated that there is no relationship between C-CLZ and EEG abnormalities. It is well-known that C-CLZ shows large inter-individual variability. However, there are no reports on the association between intra-individual variability and the side-effects of CLZ. Our results indicate that the patients with a high intra-individual variability in the $\mathrm{C} / \mathrm{D}$ ratio had a greater possibility of exhibiting EEG abnormalities. We recommend that clinicians should assess C-CLZ repeatedly to detect EEG abnormalities, and perhaps other side-effects of CLZ, early.

\section{Acknowledgments}

We are grateful to all the participants in this study. We thank Yumiko Akamine for measurement of the serum concentrations of CLZ.

\section{Conflicts of Interest}

The authors have no potential conflicts of interest to disclose.

\section{REFERENCES}

1. Freudenreich O, Weiner RD, McEvoy JP. Clozapine-induced electroencephalogram changes as a function of clozapine serum levels. Biol Psychiatry 1997;42:132-137.

2. Günther W, Baghai T, Naber D, Spatz R, Hippius H. EEG alterations and seizures during treatment with clozapine. A retrospective study of 283 patients. Pharmacopsychiatry 1993;26:69-74.

3. Haring C, Fleischhacker WW, Schett P, Humpel C, Barnas C, Saria A. Influence of patient-related variables on clozapine plasma levels. Am J Psychiatry 1990;147:1471-1475.

4. Welch J, Manschreck T, Redmond D. Clozapine-induced seizures and EEG changes. J Neuropsychiatry Clin Neurosci 1994;6:250-256.

5. Goyal N, Praharaj SK, Desarkar P, Nizamie H. Electroencephalographic abnormalities in clozapine-treated patients: a cross-sectional study. Psychiatry Investig 2011;8:372-376.

6. Menkes DB, Glue P, Gale C, Lam F, Hung CT, Hung N. Steady-state clozapine and norclozapine pharmacokinetics in Maori and European patients. EBioMedicine 2018;27:134-137.

7. Ng CH, Chong SA, Lambert T, Fan A, Hackett LP, Mahendran R, et al. An inter-ethnic comparison study of clozapine dosage, clinical response and plasma levels. Int Clin Psychopharmacol 2005;20:163-168.

8. Matsuda KT, Cho MC, Lin KM, Smith MW, Young AS, Adams JA. Clozapine dosage, serum levels, efficacy, and side-effect profiles: a comparison of Korean-American and Caucasian patients. Psychopharmacol Bull 1996;32:253-257.

9. Rajji TK, Mulsant BH, Davies S, Kalache SM, Tsoutsoulas C, Pollock $\mathrm{BG}$, et al. Prediction of working memory performance in schizophrenia by plasma ratio of clozapine to $\mathrm{N}$-desmethylclozapine. Am J Psychiatry 2015;172:579-585

10. Chang WH, Lin SK, Lane HY, Hu WH, Jann MW, Lin HN. Clozapine dosages and plasma drug concentrations. J Formos Med Assoc 1997;96: 599-605.

11. Rostami-Hodjegan A, Amin AM, Spencer EP, Lennard MS, Tucker GT, Flanagan RJ. Influence of dose, cigarette smoking, age, sex, and metabolic activity on plasma clozapine concentrations: a predictive model and nomograms to aid clozapine dose adjustment and to assess compliance in individual patients. J Clin Psychopharmacol 2004;24:70-78.

12. Piwowarska J, Radziwoń-Zaleska M, Dmochowska M, Szepietowska E, Matsumoto H, Sygitowicz G, et al. The usefulness of monitored therapy using Clozapine concentration in the blood serum for determining drug dose in Polish schizophrenic patients. Pharmacol Rep 2016;68:11201125 .

13. Haring C, Neudorfer C, Schwitzer J, Hummer M, Saria A, Hinterhuber $\mathrm{H}$, et al. EEG alterations in patients treated with clozapine in relation to plasma levels. Psychopharmacology (Berl) 1994;114:97-100.

14. Tang Y, Mao P, Li FM, Li W, Chen Q, Jiang F, et al. Gender, age, smoking behaviour and plasma clozapine concentrations in 193 Chinese inpatients with schizophrenia. Br J Clin Pharmacol 2007;64:49-56.

15. Molins C, Carceller-Sindreu M, Navarro H, Carmona C, Piñeiro M, Martínez E, et al. Plasma ratio of clozapine to N-desmethylclozapine can predict cognitive performance in treatment-resistant psychotic patients. Psychiatry Res 2017;258:153-157.

16. Carrillo JA, Herraiz AG, Ramos SI, Benítez J. Effects of caffeine withdrawal from the diet on the metabolism of clozapine in schizophrenic patients. J Clin Psychopharmacol 1998;18:311-316.

17. Dettling M, Sachse C, Brockmöller J, Schley J, Müller-Oerlinghausen B, Pickersgill I, et al. Long-term therapeutic drug monitoring of clozapine and metabolites in psychiatric in- and outpatients. Psychopharmacology (Berl) 2000;152:80-86.

18. Hägg S, Spigset $\mathrm{O}$, Mjörndal T, Dahlqvist R. Effect of caffeine on clozapine pharmacokinetics in healthy volunteers. Br J Clin Pharmacol 2000;49:59-63.

19. Palego L, Biondi L, Giannaccini G, Sarno N, Elmi S, Ciapparelli A, et al. Clozapine, norclozapine plasma levels, their sum and ratio in 50 psychotic patients: influence of patient-related variables. Prog Neuropsychopharmacol Biol Psychiatry 2002;26:473-480.

20. Perry PJ, Bever KA, Arndt S, Combs MD. Relationship between patient variables and plasma clozapine concentrations: a dosing nomogram. Biol Psychiatry 1998;44:733-738.

21. Schaber G, Stevens I, Gaertner HJ, Dietz K, Breyer-Pfaff U. Pharmacokinetics of clozapine and its metabolites in psychiatric patients: plasma protein binding and renal clearance. Br J Clin Pharmacol 1998;46:453459.

22. Seppälä NH, Leinonen EV, Lehtonen ML, Kivistö KT. Clozapine serum concentrations are lower in smoking than in non-smoking schizophrenic patients. Pharmacol Toxicol 1999;85:244-246.

23. Wetzel H, Anghelescu I, Szegedi A, Wiesner J, Weigmann H, Härter S, et al. Pharmacokinetic interactions of clozapine with selective serotonin reuptake inhibitors: differential effects of fluvoxamine and paroxetine in a prospective study. J Clin Psychopharmacol 1998;18:2-9.

24. Zullino DF, Delessert D, Eap CB, Preisig M, Baumann P. Tobacco and cannabis smoking cessation can lead to intoxication with clozapine or olanzapine. Int Clin Psychopharmacol 2002;17:141-143.

25. Jerling M, Merlé Y, Mentré F, Mallet A. Population pharmacokinetics of clozapine evaluated with the nonparametric maximum likelihood method. Br J Clin Pharmacol 1997;44:447-453.

26. Diaz FJ, Santoro V, Spina E, Cogollo M, Rivera TE, Botts S, et al. Estimating the size of the effects of co-medications on plasma clozapine concentrations using a model that controls for clozapine doses and confounding variables. Pharmacopsychiatry 2008;41:81-91.

27. Haring C, Meise U, Humpel C, Saria A, Fleischhacker WW, Hinterhuber H. Dose-related plasma levels of clozapine: influence of smoking behaviour, sex and age. Psychopharmacology (Berl) 1989;99(Suppl):S38-S40.

28. Ulrich S, Baumann B, Wolf R, Lehmann D, Peters B, Bogerts B, et al. Therapeutic drug monitoring of clozapine and relapse--a retrospective study of routine clinical data. Int J Clin Pharmacol Ther 2003;41:3-13.

29. Centorrino F, Price BH, Tuttle M, Bahk WM, Hennen J, Albert MJ, et al. EEG abnormalities during treatment with typical and atypical antipsychotics. Am J Psychiatry 2002;159:109-115.

30. Naber D, Leppig M, Grohmann R, Hippius H. Efficacy and adverse effects of clozapine in the treatment of schizophrenia and tardive dyskinesia--a retrospective study of 387 patients. Psychopharmacology (Berl) 1989;99(Suppl):S73-S76.

31. Bondesson U, Lindström LH. Determination of clozapine and its Ndemethylated metabolite in plasma by use of gas chromatography-mass 
spectrometry with single ion detection. Psychopharmacology (Berl) 1988;95:472-475.

32. Légaré N, Grégoire CA, De Benedictis L, Dumais A. Increasing the clozapine: norclozapine ratio with co-administration of fluvoxamine to enhance efficacy and minimize side effects of clozapine therapy. Med Hypotheses 2013;80:689-691.

33. Anderson SG, Livingston M, Couchman L, Smith DJ, Connolly M,
Miller J, et al. Sex differences in plasma clozapine and norclozapine concentrations in clinical practice and in relation to body mass index and plasma glucose concentrations: a retrospective survey. Ann Gen Psychiatry 2015;14:39.

34. Rajkumar AP, Poonkuzhali B, Kuruvilla A, Jacob M, Jacob KS. Clinical predictors of serum clozapine levels in patients with treatment-resistant schizophrenia. Int Clin Psychopharmacol 2013;28:50-56. 